\section{Efectos de las estatinas en cáncer: ¿potencial rol en terapéutica y prevención?}

\author{
MAURICIO CUELLO F. ${ }^{1}$, SUMIE KATO C. ${ }^{1, a}$, \\ DANIELA DÍAZ S. ${ }^{1, \mathrm{~b}}$, GARETH OWEN ${ }^{2, \mathrm{c}}$
}

\section{Effects of statins in cancer}

This review explores the evidence supporting a potential benefit of statins in cancer. In particular, the lipophilic forms (i.e. lovastatin, simvastatin, or similar) would have a therapeutic but not a preventive role. The pleiotropic effects that statins possess mainly explain this phenomenon, influencing the natural history of disease and the response to currently available therapies. By inhibiting the mevalonate pathway, statins would have a systemic effect, similar to that observed in atherosclerosis, reducing the inflammatory stimuli present in the tumor micro-environment and inhibiting the activation of intracellular signaling cascades critical for proliferation, migration/invasion and metastasis of the cancer cell. Despite all this evidence, randomized trials are needed to confirm the benefit of statins on cancer, before promoting their widespread use as a therapeutic or preventive strategy for this condition.

(Rev Med Chile 2013; 141: 227-236).

Key words: Hydroxymethylglutaryl-Co A reductase inhibitors; Lovastatin; Mevalonic acid; Neoplasms; Simvastatin.

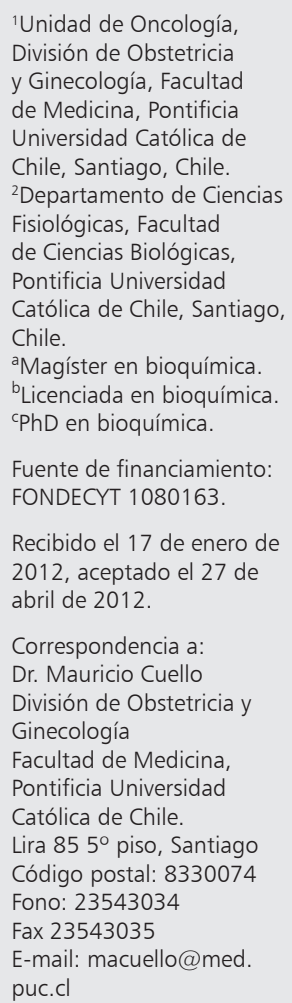

$\mathrm{E}$ $\mathrm{n}$ el mundo, millones de pacientes reciben estatinas diariamente con la finalidad de corregir el trastorno del metabolismo del colesterol que les aqueja (ej. hipercolesterolemia) y de prevenir los eventos cardiovasculares adversos asociados (ej. angina/infarto, accidente cerebrovascular, etc.). Tal intervención médica se basa en numerosos ensayos clínicos aleatorios que demuestran, por una parte, como el consumo de estatinas, como un complemento a la dieta, es efectivo en corregir el trastorno en pacientes con hipercolesterolemia y otras formas mixtas de dislipidemia, reduciendo los niveles elevados de colesterol total, de colesterol-LDL, en menor grado los de triglicéridos y apolipoproteína-B; y elevando los niveles de colesterol-HDL ${ }^{1,2}$. Junto a la capacidad de modificar el perfil lipídico, estudios in vitro y en animales, demuestran que las estatinas poseen efectos pleiotrópicos, independientes de la síntesis de colesterol, los cuales afectan la homeostasis del endotelio vascular y modifican la historia natural de la formación de la placa de ateroma ${ }^{3}$. Ello se traduce en términos prácticos, en una forma efectiva de prevención tanto para pacientes hipercolesterolémicos quienes son asintomáticos para enfermedad cardiovascular (prevención primaria) como para aquellos que sí han presentado un cuadro clínico relacionado (prevención secundaria) $)^{1,4-7}$.

En la actualidad es universalmente aceptado que la introducción de las estatinas al arsenal terapéutico ha sido uno de los factores que ha contribuido a la reducción de la mortalidad atribuible a enfermedades cardiovasculares ${ }^{8}$. En Chile, como en la mayoría de los países desarrollados, se observa una reducción paulatina en el número de dichas muertes, en ambos sexos. La estadística más reciente publicada por el INE informa una reducción de 3\% en las muertes por patología cardiovascular en la población masculina y de $7 \%$ en la femenina ${ }^{9}$. Es posible que parte de dicha reducción también se relacione al uso de estatinas.

Es importante enfatizar que los efectos de las estatinas no se limitarían a patologías que afectan el sistema cardiovascular. Estudios observacionales sugieren que las estatinas, a través de la 
reducción de los niveles de colesterol y sus efectos pleiotrópicos, afectarían de manera positiva la evolución de una serie de otras condiciones, dentro de las cuales se incluyen: la enfermedad de Alzheimer, la osteoporosis, la falla cardiaca y la enfermedad renal crónica ${ }^{10-12}$. Sin embargo, tal perfil beneficioso de las estatinas no sería tan claro para otras condiciones, en particular para el cáncer, donde la exposición prolongada a su uso pudiese asociarse a un aumento en la incidencia y un peor pronóstico de tal condición ${ }^{13}$.

La presente revisión pretende resumir la información disponible sobre los efectos que tendrían las estatinas en la incidencia de cáncer, su comportamiento biológico y su respuesta a las terapias disponibles. Para realizar esta revisión efectuamos una búsqueda en la literatura usando las bases de datos Medline y Ovid, incluyendo todos los trabajos que reunían los criterios de búsqueda y que estaban disponibles al momento de ejecutarla. Los siguientes términos fueron usados: cholesterol, HMGCoA reductase inhibitors, statins (incluyendo distintas formas ej. lovastatin, simvastatin, atorvastatin, rosuvastatin, etc) y cáncer. También incluimos en la definición los cánceres más frecuentes: mama, pulmón, próstata, estómago, colon y los cánceres ginecológicos (ej. ovario). Los términos MESH utilizados fueron 'neoplasm' e 'hydroxymethylglutaryl-CoA reductase inhibitor'. Se incluyeron trabajos publicados en inglés y español, realizados en humanos y también conducidos in vitro (cultivos celulares de tumor) y en modelos animales. Incluimos además resultados obtenidos por nuestro grupo que avalan algunos de los efectos de las estatinas (datos ya publicados y algunos no publicados).

Creemos que es importante para los médicos que prescriben estatinas el conocer los beneficios y riesgos potenciales del uso crónico de éstas, y en particular sus efectos en cáncer. Más aun cuando nuestra población envejece, se expone a factores de riesgo predisponentes (ej. obesidad, tabaquismo), parte de ella usa estatinas y cuando cada vez hay mayor incidencia/mortalidad por cáncer en Chile.

\section{Bases moleculares del efecto benéfico de las estatinas en patología cardiovascular}

Hoy en día la ateroesclerosis se considera una enfermedad inflamatoria crónica, que cursa en etapas y donde se desarrollan placas de ateroma en la capa interna de las arterias. El depósito anormal de lípidos en la pared arterial se origina en una respuesta anormal del endotelio a estímulos irritativos presentes en la circulación (ej. hipercolesterolemia, mediadores pro-inflamatorios, hipertensión, etc.). La inestabilidad de la placa predispone a eventos trombogénicos y tiene como consecuencia natural la ocurrencia de eventos coronarios agudos, accidentes cerebrovasculares y enfermedad vascular ${ }^{3,14}$.

Los efectos benéficos cardiovasculares de las estatinas se sustentan en la inhibición de la enzima 3-hidroxi-3-metilglutaril-coenzima A (HMG-CoA) reductasa, enzima crítica en la síntesis del colesterol. Dicha enzima cataliza la conversión de HMGCoA a mevalonato, un metabolito clave en la síntesis del colesterol (Figura 1). A consecuencia de esta inhibición, se produce una reducción en los niveles de colesterol total y de colesterol-LDL, dos metabolitos íntimamente relacionados con la génesis de la ateroesclerosis y el riesgo cardiovascular. Independiente de los efectos en el perfil lipídico, las estatinas ofrecen beneficio cardiovascular adicional gracias a que poseen efectos celulares conocidos como 'pleiotrópicos', los cuales impactan en la homeostasis de la pared arterial ${ }^{2}$. Tales efectos incluyen entre otros: el aumento en la expresión y actividad de la óxido nítrico sintetasa (eNOS) en células endoteliales, reducción en la migración y proliferación de células musculares lisas vasculares, inhibición de la adhesión y activación plaquetaria, reducción en la acumulación de colesterol, proliferación y activación de monocitos/macrófagos atraídos al sitio de injuria endotelial, reducción en la producción de factores quimiotácticos (i.e. MCP-1) y de metaloproteinasas, e incremento en la movilización de células madres ${ }^{2,3,15,16}$. Estos efectos se originan debido a que las estatinas generan interferencia en la formación de isoprenoides a partir de mevalonato ${ }^{1,2,16}$. Estos isoprenoides incluyen dos formas predominantes, el geranilgeranil-pirofosfato (GPP) y el farnesil-pirosfosfato (FPP). Ambas formas son críticas en la generación de cambios post-transduccionales (fenómeno denominado 'prenilación') que afectan a la unidad gamma de proteínas $G$ heterotriméricas y a pequeñas proteínas $\mathrm{G}$ monoméricas con capacidad de unir e hidrolizar GTP (GTPasas) ${ }^{2,16}$. La reducción en la prenilación de dichas proteínas afecta su anclaje a la membrana, la funcionalidad del receptor 


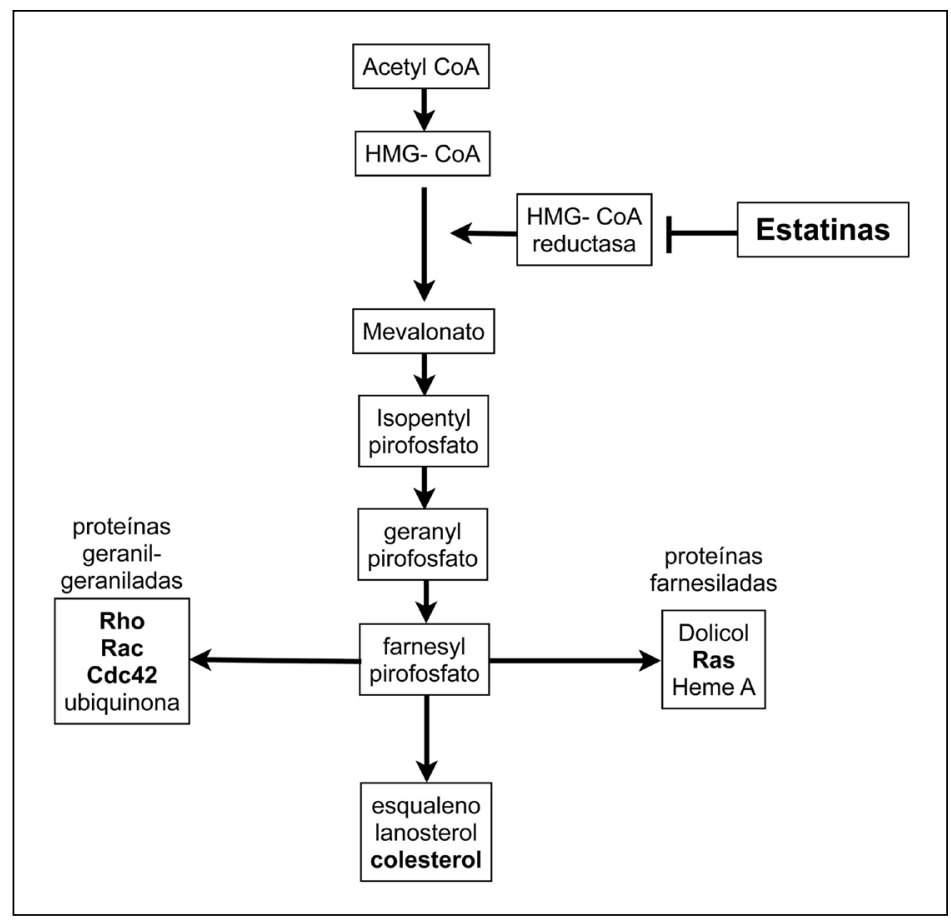

Figura 1. Esquema que resume la vía del mevalonato y el mecanismo de acción de las estatinas. Las estatinas al inhibir la enzima HMGCoA reductasa determinan la reducción en la síntesis de colesterol y de productos necesarios para la prenilación de proteínas que participan de la señalización intracelular. al cual se encuentran acopladas y la cascada de señalización intracelular que habitualmente gatillan. Dentro de dichas proteínas se encuentran las pertenecientes a la super familia de Ras (Ras, Rho, Rab, Arf y Ran). En particular, la inhibición de la vía Rho/ROCK, inducida por estatinas, afectaría la función endotelial a través del incremento en la expresión de la eNOS y de la restauración de su actividad ${ }^{16}$. Mecanismos complementarios a través de los cuales las estatinas afectarían la función endotelial incluyen la activación de la vía PI3K/ Akt, la cual induciría angiogénesis, a través de la movilización de células progenitoras endoteliales circulantes ${ }^{16}$. En conjunto, los efectos celulares gatillados en el endotelio, en células musculares lisas vasculares, en monocitos/macrófagos y plaquetas modificarían la historia natural que caracteriza la formación de la placa de ateroma y que condiciona los eventos cardiovasculares adversos ${ }^{16}$.

\section{Inflamación crónica, nexo entre ateroesclerosis y el rol de las estatinas en cáncer}

Las lesiones de la enfermedad ateroesclerótica representan una serie de respuestas celulares y moleculares específicas que corresponden a un proceso inflamatorio. La estría grasa, la más precoz de las lesiones en la evolución natural de la enfermedad, es una lesión inflamatoria pura, constituida sólo por macrófagos derivados de monocitos y linfocitos- $\mathrm{T}^{17}$. Varias líneas de investigación sustentan que la inflamación y eventualmente la infección crónica, por diversos mecanismos, contribuirían no sólo al inicio sino a la progresión de la ateroesclerosis. Más aun, la evidencia disponible apoya que la inflamación arterial jugaría un rol crucial, vinculando aterogénesis y trombogénesis, en pacientes con síndromes coronarios agudos ${ }^{2,3,14}$. Interesantemente, la concepción actual en relación a cáncer, otorga a esta enfermedad características comunes a las observadas en la ateroesclerosis, donde la inflamación también tendría un papel central. Es así como evidencia epidemiológica y experimental apoya que la presencia de una respuesta inflamatoria crónica, constituye un pilar fundamental en la génesis, progresión y agresividad biológica de diversos cánceres epiteliales y de otras estirpes ${ }^{18-20}$. A modo de ejemplo, tanto el cáncer de colon como el cáncer de esófago han sido vinculados en su génesis y evolución a condiciones que se asocian a una respuesta inflamatoria crónica anormal ${ }^{18,19}$. Tal es el caso de la enfermedad infla- 
matoria intestinal y el esófago de Barret, ambas condiciones donde es posible observar histológicamente signos de inflamación crónica, y las cuales se asociarían a un mayor riesgo de cáncer colorectal y de adenocarcinoma de esófago, respectivamente. En ambos casos, las lesiones pre-malignas serían lesiones inflamatorias, ya que el microambiente del tumor contendría una variedad de leucocitos $\mathrm{y}$ factores inflamatorios ${ }^{18,19}$.

Es interesante mencionar que, dentro de los factores de riesgo para ciertas neoplasias se encuentran elementos comunes a los que condicionan el riesgo de ateroesclerosis. Tal es el caso de la obesidad, tabaquismo y dislipidemias, todas condiciones en las cuales se ha logrado evidenciar la presencia de un ambiente pro-inflamatorio sistémico, a través de la medición de niveles de interleuquinas (ej. IL-6), proteína C reactiva (PCR) y TNFalfa ${ }^{15,18,19,21}$. Dichos marcadores, asociados a riesgo cardiovascular, también se relacionarían con el riesgo y pronóstico de algunas neoplasias (ej. colon, pulmón, ovario) ${ }^{22,23}$. El ambiente inflamatorio afectaría de manera especial a tejidos susceptibles donde podría generarse una neoplasia. $\mathrm{Al}$ igual que en la ateroesclerosis, el modelo de inflamación no se circunscribiría a un sólo tipo celular (ej. células endoteliales) ${ }^{18-20}$. El cáncer, actualmente, ya no se considera como el crecimiento anormal de una estirpe clonal celular, sino que es el resultado de la interacción de dicha estirpe con su medio ambiente, en donde se incluye la participación de otros tipos celulares, a fin de permitir el crecimiento y expansión tumoral. Así en la carcinogénesis, crecimiento tumoral y metástasis participan tanto la célula cancerosa en sus distintas etapas evolutivas (célula iniciadora, células en transición epitelio-mesenquimal, célula metastásica/resistente, etc.) como también las células inflamatorias (monocitos/macrófagos activados) infiltrando el tumor, las células del estroma local que reaccionan a su presencia, y las células madres ahí presentes, y que estimuladas, adquieren la capacidad de diferenciación (ej. células progenitoras con capacidad de angiogénesis, etc. $)^{20}$. Al igual que en la ateroesclerosis, la presencia de un agente pro-inflamatorio afecta el comportamiento de los distintos componentes celulares presentes en el microambiente tumoral y las interacciones que entre ellos se producen. De hecho, muchas de las cascadas de señalización activas en la ateroesclerosis se encuentran gatilladas de manera exagerada en cáncer. Dichas cascadas permiten la proliferación descontrolada, la capacidad de supervivencia a la acción de agentes o condiciones que normalmente inducirían apoptosis, la adquisición de resistencia, la adaptación metabólica, la capacidad de inhibir el reconocimiento por parte del sistema inmune, por nombrar algunas de ellas ${ }^{20}$. Tal como se muestra en la Figura 2, la presencia de una condición sistémica pro-inflamatoria, como ocurre en pacientes con hipercolesterolemia o con obesidad, donde frecuentemente los niveles séricos de PCR o IL-6 se encuentran elevados, favorecería la activación exagerada de dichas vías de señalización ${ }^{24}$. Uno de los mecanismos que explicaría tal activación, serían los cambios en la composición de la membrana celular, inducidos por el exceso de colesterol, lo cual modificaría la constitución de las balsas lipídicas y caveolas, lugar donde se congregan receptores que gatillan vías tanto de supervivencia (ej. el receptor del factor de crecimiento epidérmico EGFR) como de muerte celular (ej. TRAIL, un miembro de la familia de receptores TNF) ${ }^{25}$. Otro mecanismo explicativo vincularía a los mediadores pro-inflamatorios y los niveles elevados de leptina, presentes en pacientes obesos, los cuales podrían favorecer el estímulo, interacción cruzada y amplificación de señales mediada por receptores trans-membrana (por ej. entre los receptores ObR, gatillados por leptina, y los miembros de la familia EGF, activados por EGF, cuya señal sería amplificada al converger ambos en la misma vía PI3/AKT kinasa, favoreciendo la proliferación y supervivencia). Tal desbalance promovería el comportamiento más agresivo de diversos cánceres epiteliales (ej. cáncer de próstata) ${ }^{26,27}$.

\section{Efectos de las estatinas en cáncer}

Los efectos potenciales de las estatinas en cáncer pueden agruparse en: a) el impacto que el uso de estatinas tendría en el riesgo de desarrollar cáncer; b) en el comportamiento biológico del cáncer y c) los efectos que ellas tendrían cuando se asocian con terapias disponibles para el cáncer (ej. quimioterapia) y en el riesgo de recurrencia futura.

\section{a) Riesgo de cáncer en pacientes usuarios(as) de estatinas}

A mediados de la década 1990-99, estudios realizados en modelos animales y datos provenientes de estudios epidemiológicos despertaron 
Rol potencialmente benéfico de las estatinas en cáncer - M. Cuello et al

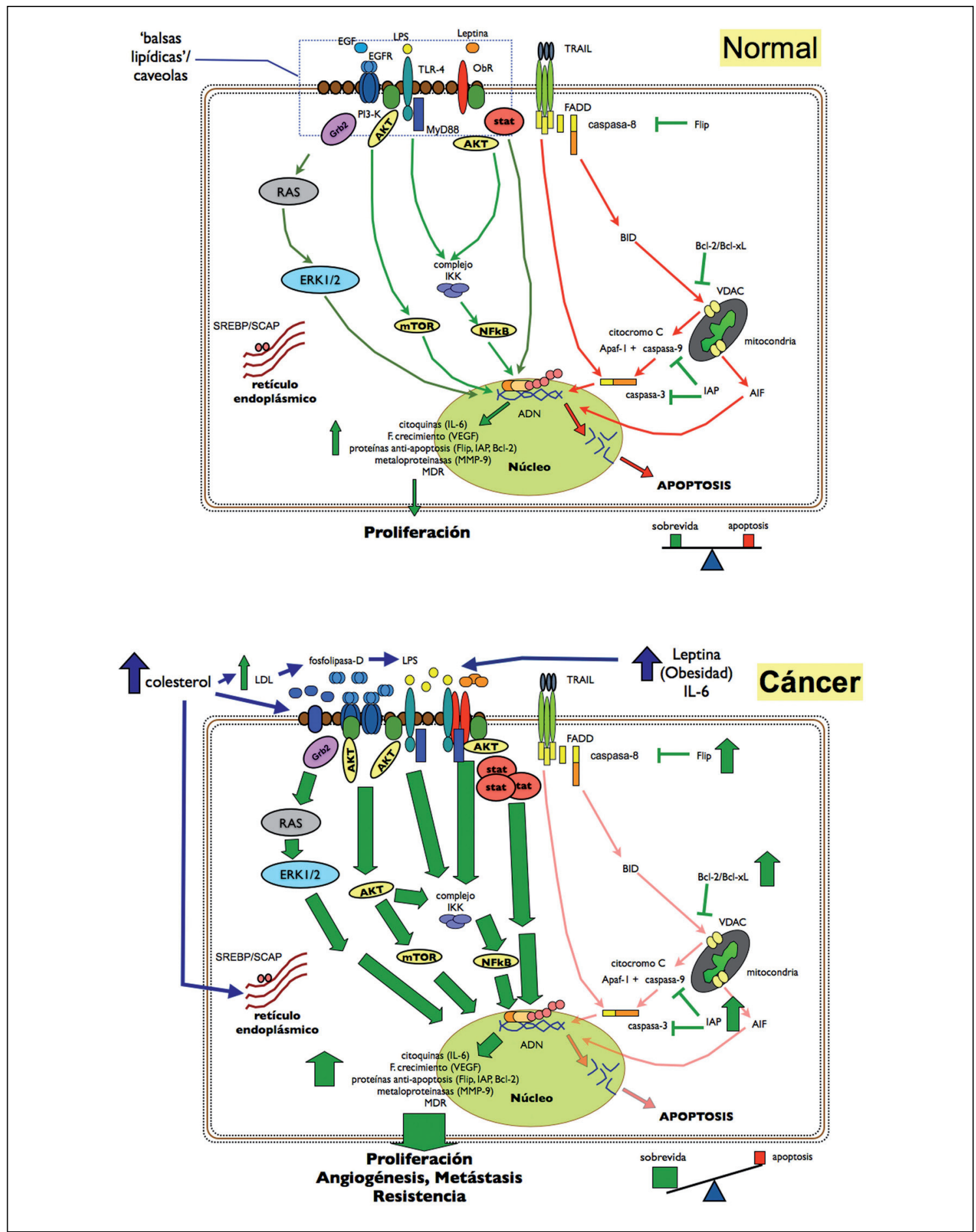

Figura 2. Esquema comparativo de las vías de señalización entre una célula normal y una cancerosa. En la célula cancerosa coexisten el aumento en la expresión de receptores que promueven proliferación y sobrevida y hay reducción en la expresión de componentes que promueven la apoptosis. En presencia de agentes pro-inflamatorios (ej. IL-6, niveles altos de colesterol, leptina) en el medio ambiente, las vías de supervivencia se gatillan y activan de manera exagerada. 
la inquietud sobre si el uso prolongado de estatinas podría aumentar la incidencia de cáncer. Dentro de dichos estudios, algunos asociaban la reducción en los niveles del colesterol, producto del uso de estatinas, con mayor incidencia de carcinoma hepatocelular, adenoma pulmonar, cáncer de tiroides y linfomas ${ }^{13}$. Mecanismos moleculares potenciales que apoyaban dicho efecto deletéreo incluían: la estimulación de la vía de señalización de TGF $\beta$ mediada por niveles reducidos de colesterol, lo cual podría favorecer el desarrollo de carcinoma renal; la asociación de algunas formas de estatinas (ej. lovastatina) con una mayor incidencia de anomalías mitóticas las cuales afectarían el desarrollo y la función de los centrómeros, aumentando el riesgo de mutaciones y de transformación maligna; la inhibición del promotor tipo IV del transactivador clase II del complejo de histocompatibilidad mayor, lo cual afectaría la función y reconocimiento antigénico por parte de los leucocitos, con la consiguiente inmunosupresión; y la inducción de angiogénesis por aumento en la expresión y actividad de la eNOS $^{13,26}$. Cabe señalar, eso sí, que varios de dichos efectos fueron observados en modelos animales utilizando concentraciones muy superiores a las indicadas en pacientes con hipercolesterolemia ${ }^{13}$. Tal evidencia llevó a varios autores a revisar, de manera reiterada, tanto los estudios aleatorios como observacionales existentes que evaluaban el rol de las estatinas en prevención cardiovascular o tratamiento de la hipercolesterolemia, a fin de poder confirmar o descartar dicho temor. Dentro de ellas, las revisiones más recientes, que incluyen una revisión sistemática, algunos meta-análisis y estudios de caso-control anidados, establecen que no hay evidencia concluyente que apoye un riesgo mayor de desarrollar cáncer en pacientes recibiendo estatinas por períodos de hasta 4-6 años. Tampoco demuestran fehacientemente que exista un aumento en la incidencia de un cáncer específico ${ }^{28-30}$. Más aun, algunos de estos estudios otorgarían un rol benéfico al uso de estatinas en la prevención y desarrollo de ciertas formas más agresivas de cáncer (ej. cáncer de próstata, colon) ${ }^{30,31}$.

\section{b) Efectos de las estatinas en el comportamiento biológico del cáncer}

Estudios in vitro y en modelos animales apoyan que los efectos de las estatinas en ateroesclerosis, tanto los dependientes de la síntesis de colesterol como los pleiotrópicos, también tendrían un rol en la historia natural de algunos cánceres ${ }^{26,32,33}$.

En estudios anteriores, los autores de este trabajo, así como otros, han demostrado que algunos cánceres serían dependientes de la actividad de la enzima HMGCoA reductasa y de la vía del mevalonato (ej. cáncer de colon y próstata) ${ }^{26,32,33}$. En cánceres ginecológicos, y en particular, el cáncer de ovario, se ha encontrado, tanto en líneas celulares como en cultivos primarios, niveles elevados de expresión de esta enzima comparado con la contraparte benigna ${ }^{32}$. Para otros cánceres tal expresión se correlacionaría con la respuesta terapéutica y el pronóstico de la enfermedad ${ }^{34-36}$. Tal como lo muestra la Figura 3, la incubación con estatinas lipofílicas (ej. lovastatina o simvastatina) induce inicialmente autofagia, probablemente como un mecanismo adaptivo, fenómeno dependiente de la dosis y del tiempo de exposición (observaciones no publicadas). Tiempos más prolongados de exposición determinan la muerte celular ${ }^{32}$. Como explicación a este fenómeno, en los cánceres antes mencionados, en cultivos celulares se observa inactivación de cascadas que son esenciales para la supervivencia de la célula cancerosa (por ej. la vía $\mathrm{PI} 3 \mathrm{~K} / \mathrm{Akt} / \mathrm{mTOR}$ o la vía ras/MEK/ERK1/2) ${ }^{26,32}$. La inhibición de la vía PI3K/Akt sería consecuencia de la depleción de colesterol en la membrana inducida por estatinas. Ello alteraría la constitución de los microdominios de la membrana celular, tanto balsas lipídicas como caveolas, afectando la localización, funcionalidad y acoplamiento de los receptores que gatillan esta vía de señalización (ej. EGF $)^{26}$. La dependencia en la vía del mevalonato se confirma al demostrar que la suplementación con geranylgeranyl-pirofosfato (metabolito ubicado río abajo en la cadena de síntesis de mevalonato) revierte de manera casi completa tanto la autofagia como la apoptosis inducida por estatinas ${ }^{32}$.

Un hallazgo relevante de los autores de este trabajo es el hecho de que son las estatinas lipofílicas y no las hidrofilicas (por ej. pravastatina) las que ejercen los efectos sobre cánceres dependientes de la enzima HMGCoA reductasa ${ }^{32}$. Este hallazgo, sumado al hecho de que no todos los cánceres dependen metabólicamente de los niveles de expresión de HMGCoA reductasa, da una explicación biológica a las disparidades encontradas en estudios observacionales ${ }^{34,35}$. Al momento de realizar dichos estudios no se conocía sobre la variabilidad en la expresión de HMGCoA por 


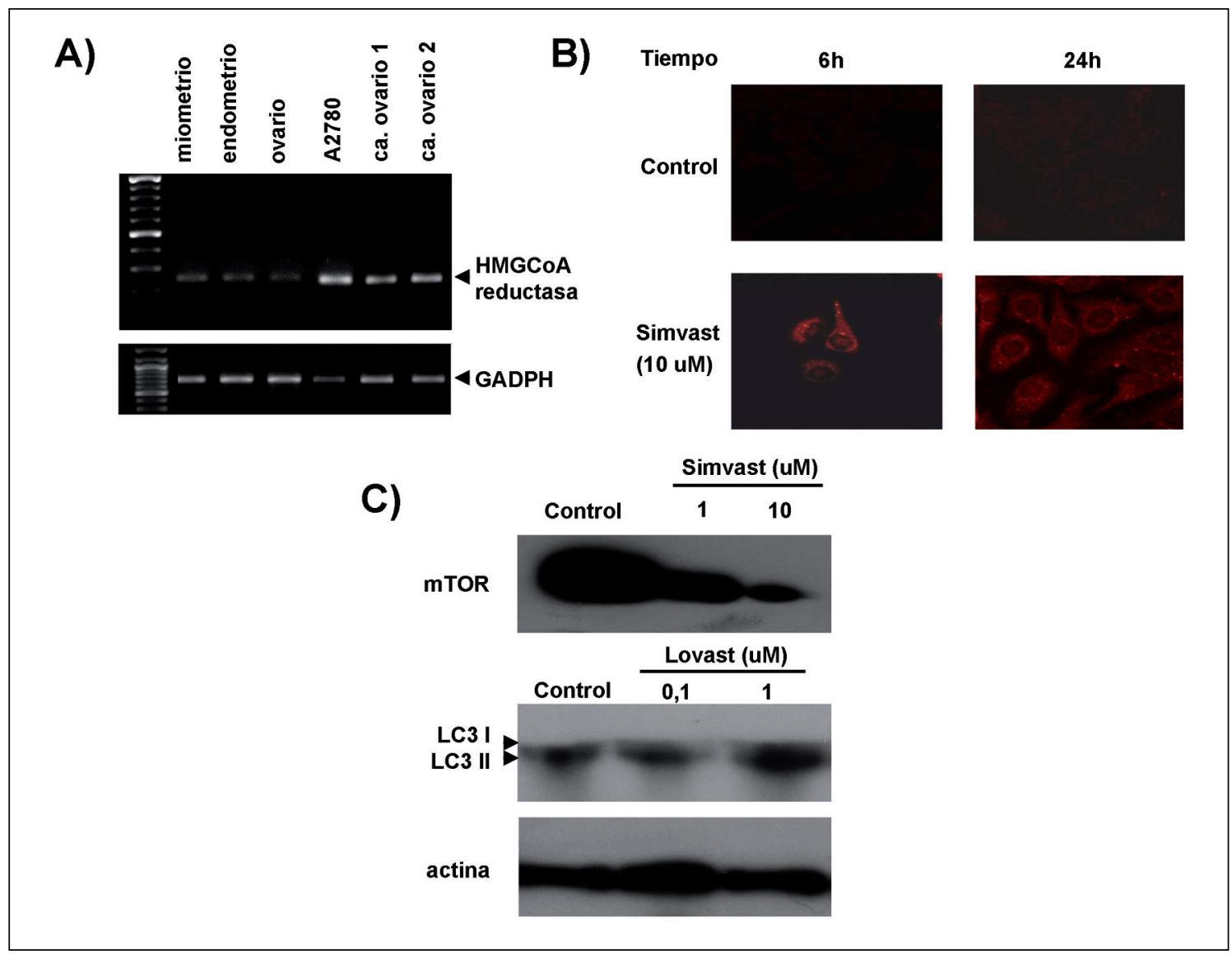

Figura 3. La exposición a estatinas (simvastatina y lovastatina) induce autofagia por parte de células cancerosas de ovario. En panel A se compara la expresión de HMGCoA reductasa entre cultivos primarios de tejidos normales (miometrio, endometrio y epitelio ovárico) y cancerosos (A2780, ca. ovario 1 y 2) medidos por RT-PCR (GADPH aparece como control de carga). En los paneles $B$ y $C$, se muestran evidencias de la autofagia inducida por estatinas a través de inmunocitología (usando marcador de autofagia LC3 II, color rojo, aumento 100X, panel B) y por western blot (medido por reducción en la expresión de mTOR y el incremento en banda LC3II, actina aparece como control de carga, panel C).

parte de distintos cánceres ni del efecto variable que distintas formas y dosis de tratamiento de las estatinas tenían sobre ellos.

Los efectos de las estatinas no se limitarían a la inducción de muerte celular. Las estatinas, a través de sus efectos pleiotrópicos no sólo afectarían a las células cancerosas, sino también a aquellas que interactúan y que forman parte del microambiente tumoral (ej. células inmunes que infiltran el tumor, células progenitoras vasculares, células madres $)^{37}$. La Tabla 1 resume los mecanismos a través de los cuales las estatinas ejercerían su mecanismo antitumoral. Dentro de dichos efectos se incluyen: la inhibición de la proliferación celular, la promoción de apoptosis, la inhibición de la angiogénesis, la modulación de la respuesta inmune e inflamatoria a nivel local y sistémico y la reducción en la capacidad de invasión y de metástasis $^{13,26,29,33,37}$.

\section{c) Efectos de la asociación de estatinas a terapias actualmente usadas en cáncer}

Estudios in vitro y en modelos animales apoyan que la asociación de estatinas a algunas quimioterapias (ej. cisplatino, doxorubicina) e inmunoterapias (ej. trastuzumab, gefitinib) resultaría en una mayor respuesta terapéutica por parte de algunos cánceres (ej. tumores gastrointestinales, mama, pulmón $)^{38-40}$. Los autores, tal como muestra la Figura 4, han demostrado la 
Tabla 1. Mecanismos moleculares que explican el efecto potencialmente benéfico de las estatinas en cáncer

\begin{tabular}{|c|c|}
\hline 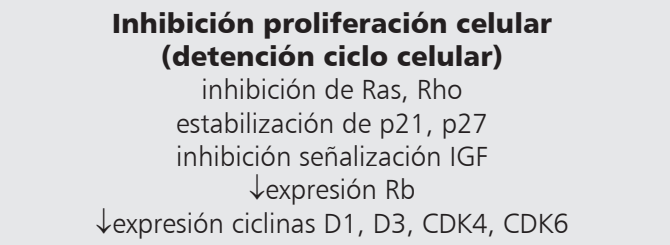 & $\begin{array}{c}\text { Inhibición de la señalización vía } \\
\text { balsas lipídicas/caveolas } \\
\text { inhibición de señalización receptor EGF } \\
\text { inhibición cascada PI3K/Akt }\end{array}$ \\
\hline 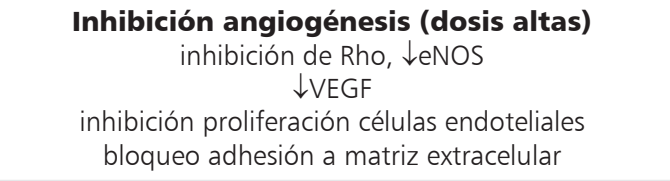 & 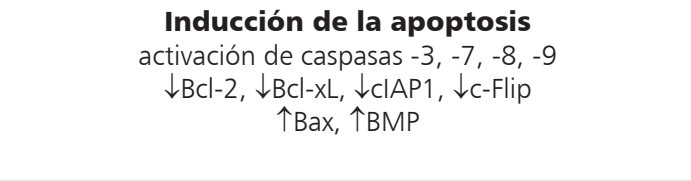 \\
\hline 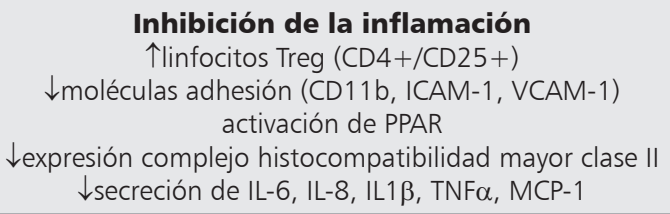 & 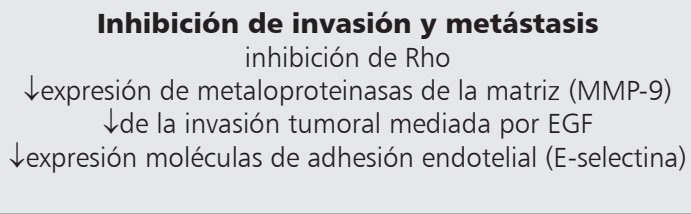 \\
\hline
\end{tabular}

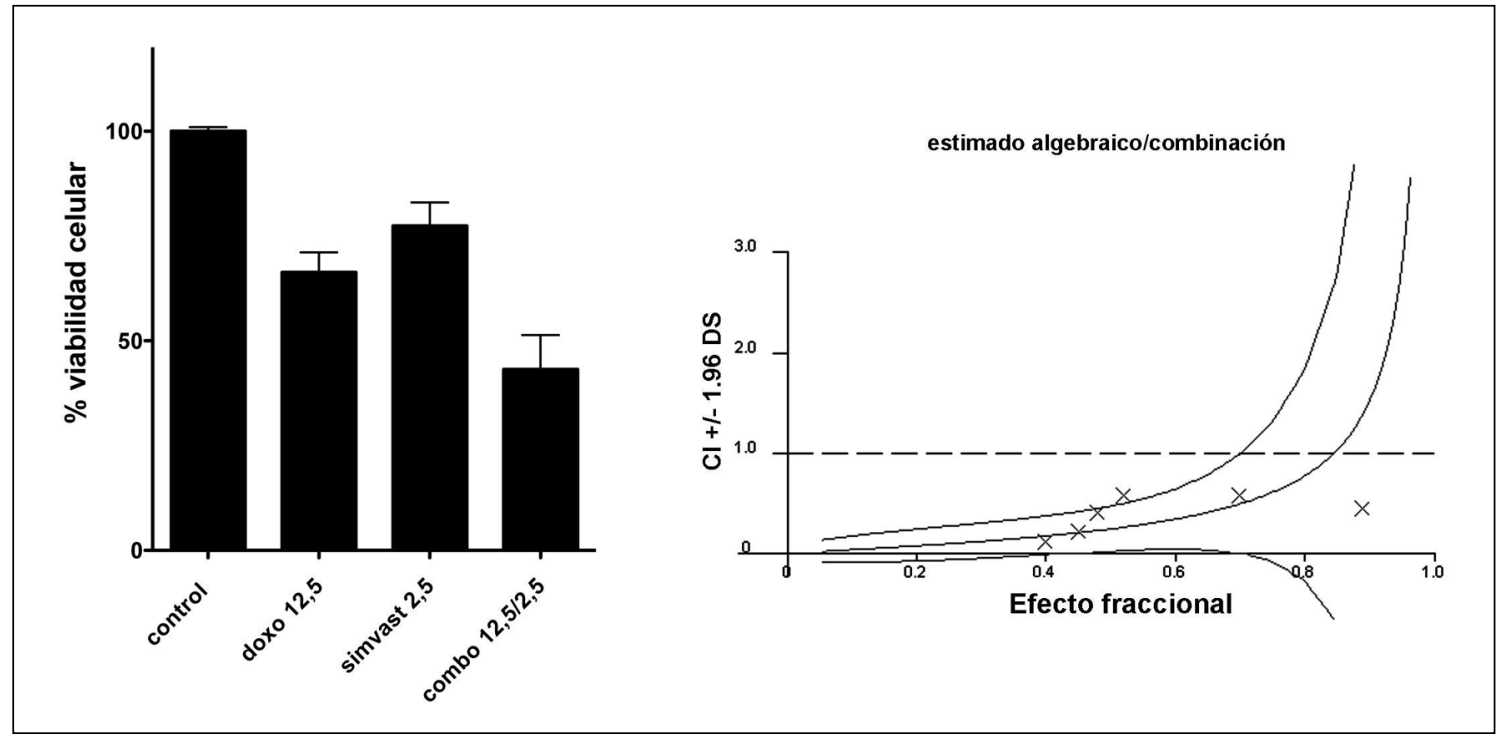

Figura 4. Efecto sinérgico de las estatinas con quimioterapia (ej. simvastatina 2,5 uMy doxorubicina 12,5 uM por 24 h) en células de cáncer de ovario (línea A2780). El análisis isobolograma confirmó el sinergismo. Cl: coeficiente de interacción, un valor $<1$ indica sinergismo.

inducción sinérgica de citotoxicidad con el uso de estatinas y quimioterapia en cáncer de ovario ${ }^{32}$. Tal efecto explica, en parte, el hallazgo de un estudio retrospectivo reciente donde pacientes con cáncer de ovario, tratadas con cirugía y quimioterapia, y que recibían concomitantemente estatinas como prevención cardiovascular, experimentaron mejor sobrevida que aquellas que no las recibieron ${ }^{41}$. Tal beneficio se debería a la inducción de apoptosis por las estatinas y a la sensibilización a quimioterapia al modificar la funcionalidad de los receptores que se encargan de la entrada y salida del fármaco desde la célula ${ }^{42}$.

Apoyando lo antes mencionado, estudios de cohorte en pacientes tratados por cáncer de próstata y mama sugerirían la existencia de un 
Rol potencialmente benéfico de las estatinas en cáncer - M. Cuello et al

beneficio en sobrevida y riesgo de recurrencia, ya sea con el uso exclusivo de estatinas o en asociación con otras terapias ${ }^{43-45}$.

\section{Conclusiones}

La evidencia actualmente disponible, tanto en modelos in vitro como in vivo, sustenta un rol más bien benéfico que dañino para las estatinas en varios cánceres. Dicho efecto dependería del tipo de estatina utilizada, de la dosis administrada y del tipo de cáncer. La inhibición de la vía del mevalonato, al reducir la síntesis de colesterol e impedir la prenilación de proteínas críticas para la señalización intracelular de la célula cancerosa, asociado a la reducción del ambiente proinflamatorio que caracteriza esta condición, sería el mecanismo principal detrás de dicho efecto. Aunque los resultados de este tipo de estudios son alentadores, la interpretación de dichos hallazgos posee las limitaciones propias del modelo de estudio y por ende deben necesariamente ser corroborados en estudios clínicos aleatorios efectuados en humanos. Por tanto, consideramos que no existe aún suficiente evidencia que avale el uso rutinario de estatinas, tanto en prevención como terapia, fuera del contexto de protocolos clínicos. En la actualidad existen una serie de estudios en curso que pretenden confirmar el rol de las estatinas en cáncer.

\section{Referencias}

1. Last AR, Ference JD, Falleroni J. Pharmacologic treatment of hyperlipidemia. Am Fam Physician 2011; 84: 551-8.

2. Mennickent CS, Bravo DM, Calvo MC, Avello LM. [Pleiotropic effects of statins]. Rev Med Chile 2008; 136: 775-82.

3. Libby P, Ridker PM, Hansson GK. Progress and challenges in translating the biology of atherosclerosis. Nature 2011; 473: 317-25.

4. Tonelli M, Lloyd A, Clement F, Conly J, Husereau D, Hemmelgarn B, et al. Efficacy of statins for primary prevention in people at low cardiovascular risk: a metaanalysis. CMAJ 2011; 183: E1189-202.

5. Kostapanos MS, Elisaf MS. JUPITER and satellites: Clinical implications of the JUPITER study and its secondary analyses. World J Cardiol 2011; 3: 207-14.

6. Pande RL, Perlstein TS, Beckman JA, Creager MA. Se- condary prevention and mortality in peripheral artery disease: National Health and Nutrition Examination Study, 1999 to 2004. Circulation 2011; 124: 17-23.

7. Pflieger M, Winslow BT, Mills K, Dauber IM. Medical management of stable coronary artery disease. Am Fam Physician 2011; 83: 819-26.

8. Lewis SJ. Lipid-lowering therapy: who can benefit? Vasc Health Risk Manag 2011; 7: 525-34.

9. Villalón G, Ghio G, Vera S. Evolución de la mortalidad en Chile según causas de muerte y edad, 1990-2007. In. Santiago: INE; 2010. p. 1-59.

10. King A. Prevention: Statins go beyond cardioprotection. Nat Rev Cardiol 2011; 8: 609.

11. Pac-Soo C, Lloyd DG, Vizcaychipi MP, Ma D. Statins: the role in the treatment and prevention of Alzheimer's neurodegeneration. J Alzheimers Dis 2011; 27: 1-10.

12. Uzzan B, Cohen R, Nicolas P, Cucherat M, Perret GY. Effects of statins on bone mineral density: a metaanalysis of clinical studies. Bone 2007; 40: 1581-7.

13. Gonyeau MJ, Yuen DW. A clinical review of statins and cancer: helpful or harmful? Pharmacotherapy 2010; 30: 177-94.

14. Lusis AJ. Atherosclerosis. Nature 2000; 407: 233-41.

15. Balanescu S, Calmac L, Constantinescu D, Marinescu M, Onut R, Dorobantu M. Systemic inflammation and early atheroma formation: are they related? Maedica (Buchar) 2010; 5: 292-301.

16. Zhou Q, Liao JK. Pleiotropic effects of statins. - Basic research and clinical perspectives. Circ J 2010; 74: 81826.

17. Ross R. Atherosclerosis, An Inflammatory Disease. New England Journal of Medicine 1999; 340: 115-26.

18. Aggarwal BB, Shishodia S, Sandur SK, Pandey MK, Sethi G. Inflammation and cancer: how hot is the link? Biochem Pharmacol 2006; 72: 1605-21.

19. Coussens LM, Werb Z. Inflammation and cancer. Nature 2002; 420: 860-7.

20. Hanahan D, Weinberg RA. Hallmarks of cancer: the next generation. Cell 2011; 144: 646-74.

21. Dobrzycka B, Terlikowski SJ, Kowalczuk O, Kinalski M. Circulating levels of TNF-alpha and its soluble receptors in the plasma of patients with epithelial ovarian cancer. Eur Cytokine Netw 2009; 20: 131-4.

22. Pine SR, Mechanic LE, Enewold L, Chaturvedi AK, Katki $\mathrm{HA}$, Zheng YL, et al. Increased levels of circulating interleukin 6, interleukin 8, C-reactive protein, and risk of lung cancer. J Natl Cancer Inst 2011; 103: 1112-22.

23. Kemik O, Sumer A, Kemik AS, Hasirci I, Purisa S, Dulger $\mathrm{AC}$, et al. The relationship among acute-phase response proteins, cytokines and hormones in cachectic patients with colon cancer. World J Surg Oncol 2010; 8: 85. 
24. Pardina E, Ferrer R, Baena-Fustegueras JA, Rivero J, Lecube A, Fort JM, et al. Only C-Reactive Protein, but not TNF-alpha or IL6, Reflects the Improvement in Inflammation after Bariatric Surgery. Obes Surg 2011.

25. Staubach S, Hanisch FG. Lipid rafts: signaling and sorting platforms of cells and their roles in cancer. Expert Rev Proteomics 2011; 8: 263-77.

26. Papadopoulos G, Delakas D, Nakopoulou L, Kassimatis T. Statins and prostate cancer: molecular and clinical aspects. Eur J Cancer 2011; 47: 819-30.

27. Paz-Filho G, Lim EL, Wong ML, Licinio J. Associations between adipokines and obesity-related cancer. Front Biosci 2011; 16: 1634-50.

28. Vinogradova Y, Coupland C, Hippisley-Cox J. Exposure to statins and risk of common cancers: a series of nested case-control studies. BMC Cancer 2011; 11: 409.

29. Boudreau DM, Yu O, Johnson J. Statin use and cancer risk: a comprehensive review. Expert Opin Drug Saf 2010; 9: 603-21.

30. Kuoppala J, Lamminpaa A, Pukkala E. Statins and cancer: A systematic review and meta-analysis. Eur J Cancer 2008; 44: 2122-32.

31. Hede K. Hints that statins reduce colon cancer risk finally being put to the test. J Natl Cancer Inst 2011; 103: 364-6.

32. Kato S, Smalley S, Sadarangani A, Chen-Lin K, Oliva B, Branes J, et al. Lipophilic but not hydrophilic statins selectively induce cell death in gynaecological cancers expressing high levels of HMGCoA reductase. J Cell Mol Med 2010; 14: 1180-93.

33. Bardou M, Barkun A, Martel M. Effect of statin therapy on colorectal cancer. Gut 2010; 59: 1572-85.

34. Brennan DJ, Laursen H, O'Connor DP, Borgquist S, Uhlen M, Gallagher WM, et al. Tumor-specific HMGCoA reductase expression in primary premenopausal breast cancer predicts response to tamoxifen. Breast Cancer Res 2011; 13: R12.

35. Brennan DJ, Brandstedt J, Rexhepaj E, Foley M, Ponten F, Uhlen M, et al. Tumour-specific HMG-CoAR is an independent predictor of recurrence free survival in epithelial ovarian cancer. BMC Cancer 2010; 10: 125.

36. Clendening JW, Pandyra A, Boutros PC, El Ghamrasni S, Khosravi F, Trentin GA, et al. Dysregulation of the mevalonate pathway promotes transformation. Proc Natl Acad Sci U S A 2010; 107: 15051-6.

37. Gauthaman K, Fong CY, Bongso A. Statins, stem cells, and cancer. J Cell Biochem 2009; 106: 975-83.

38. Budman DR, Tai J, Calabro A. Fluvastatin enhancement of trastuzumab and classical cytotoxic agents in defined breast cancer cell lines in vitro. Breast Cancer Res Treat 2007; 104: 93-101.

39. Mantha AJ, Hanson JE, Goss G, Lagarde AE, Lorimer IA, Dimitroulakos J. Targeting the mevalonate pathway inhibits the function of the epidermal growth factor receptor. Clin Cancer Res 2005; 11: 2398-407.

40. Feleszko W, Mlynarczuk I, Balkowiec-Iskra EZ, Czajka A, Switaj T, Stoklosa T, et al. Lovastatin potentiates antitumor activity and attenuates cardiotoxicity of doxorubicin in three tumor models in mice. Clin Cancer Res 2000; 6: 2044-52.

41. Elmore RG, Ioffe Y, Scoles DR, Karlan BY, Li AJ. Impact of statin therapy on survival in epithelial ovarian cancer. Gynecol Oncol 2008; 111: 102-5.

42. Goard CA, Mather RG, Vinepal B, Clendening JW, Martirosyan A, Boutros PC, et al. Differential interactions between statins and P-glycoprotein: implications for exploiting statins as anticancer agents. Int J Cancer 2010; 127: 2936-48.

43. Mondul AM, Han M, Humphreys EB, Meinhold CL, Walsh PC, Platz EA. Association of statin use with pathological tumor characteristics and prostate cancer recurrence after surgery. J Urol 2011; 185: 1268-73.

44. Gutt R, Tonlaar N, Kunnavakkam R, Karrison T, Weichselbaum RR, Liauw SL. Statin use and risk of prostate cancer recurrence in men treated with radiation therapy. J Clin Oncol 2010; 28: 2653-9.

45. Ahern TP, Pedersen L, Tarp M, Cronin-Fenton DP, Garne JP, Silliman RA, et al. Statin prescriptions and breast cancer recurrence risk: a Danish nationwide prospective cohort study. J Natl Cancer Inst 2011; 103: 1461-8. 